\title{
THE JANTZEN FILTRATION OF A CERTAIN CLASS OF VERMA MODULES
}

\author{
JONG-MIN KU
}

\begin{abstract}
Let $G=N_{+} \oplus H \oplus N_{-}$be a Kac-Moody Lie algebra. For each $M$ in the category 0 of $G$-modules, there is a filtration $\left(M_{i}\right)_{i \geq 0}$ by $G$ submodules of $M$ naturally associated with the set $\left\{v \in M \mid N_{+} v=0\right\}$. If $G$ is symmetrizable and $M$ is a Verma module, $M_{i}=M^{i}$ for all $i$ if and only if $[M: L(\mu)]=\operatorname{dim} \operatorname{Hom}_{G}(M(\mu), M)$ for all $\mu \in H^{*}$ where $\left(M^{i}\right)_{i \geq 0}$ is the Jantzen filtration of $M$. The main tools used are the nondegenerate form on each $M^{i} / M^{i+1}$ together with the $\Gamma$-operator of $G$.
\end{abstract}

1. Introduction. Let $G$ be a Kac-Moody Lie algebra over a field $\phi$ of characteristic zero. Fix a Cartan subalgebra $H$ of $G$. Let $\left\{\alpha_{1}, \ldots, \alpha_{n}\right\}$ be a simple root system of $G$ with $e_{1}, \ldots, e_{n}, h_{1}, \ldots, h_{n}, \ldots, f_{1}, \ldots, f_{n}$ as the corresponding canonical generators. As usual, $G=N_{+} \oplus H \oplus N_{-}$is a direct sum of subalgebras where $N_{+}$(resp. $N_{-}$) is the subalgebra of $G$ generated by $e_{1}, \ldots, e_{n}$ (resp. $f_{1}, \ldots, f_{n}$ ). For a Lie algebra $B$, let $U(B)$ be the universal enveloping algebra of $B$ and denote by $U(B)^{0}$ its augmentation ideal.

Let $O$ be the category of $G$-modules as defined in [1]. For each $G$-module $M \in O$, one defines a natural filtration $M \supset M_{0} \supset M_{1} \supset M_{2} \supset \cdots$ by $G$-submodules of $M$ as follows:

Definition 1.1. For a $G$-module $M \in 0, E_{0}(M)=\left\{x \in M \mid e_{i} x=0 \forall i=\right.$ $1, \ldots, n\}$. Inductively, for $k=1,2, \ldots, E_{k}(M)=U\left(N_{-}\right)^{0} E_{k-1}(M) \cap E_{0}(M)$. Then $M_{k}=U\left(N_{-}\right) E_{k}(M)$ for all $k=0,1,2, \ldots$

The interest of the above filtration lies in a conjecture of Jantzen which states that $M(\lambda)_{i} \subset M(\lambda)^{i}$ for all $i=0,1,2, \ldots$, where $M(\lambda)$ is the Verma module of highest weight $\lambda \in H^{*}$ and $M(\lambda)=M(\lambda)^{0} \supset M(\lambda)^{1} \supset M(\lambda)^{2} \supset \cdots$ is the Jantzen filtration of $M(\lambda)$. The original conjecture (cf. [2]) is stated only for finitedimensional semisimple Lie algebras. Here we have generalized it to all Kac-Moody Lie algebras. Presumably, it is not known in general how this conjecture should be properly attacked.

The purpose of this paper is to study the conjecture for a certain class of Verma modules. It turns out that the class of Verma modules considered here can be characterized by the coincidence of the two filtrations. To state the result, for a $G$-module $M \in \mathcal{O}$, denote by $[M: L(\mu)]$ the multiplicity of $L(\mu)$ in $M$ where, for $\mu \in H^{*}, L(\mu)$ is the irreducible quotient of $M(\mu)$.

THEOREM 1.2. Let $G$ be symmetrizable and $\lambda \in H^{*}$. Then $M(\lambda)_{i}=M(\lambda)^{i}$ for all $i=0,1,2, \ldots$ if and only if $[M(\lambda): L(\mu)]=\operatorname{dim}_{\operatorname{Hom}_{G}}(M(\mu), M(\lambda))$ for all $\mu \in H^{*}$.

Received by the editors December 9, 1985.

1980 Mathematics Subject Classification (1985 Revision). Primary 17B67.

(C) 1987 American Mathematical Society $0002-9939 / 87 \$ 1.00+\$ .25$ per page 
One direction of the proof of Theorem 1.2 uses the nondegenerate form on each $M(\lambda)^{i} / M(\lambda)^{i+1}$ associated with the construction of the Jantzen filtration. The other direction calls for the generalized Casimir operator, available only when $G$ is symmetrizable.

There is a rather interesting corollary to the proof of Theorem 1.2 which provides a possible inductive argument for the Jantzen conjecture:

COROllary 1.3. Let $\lambda \in H^{*}$. Suppose $M(\lambda)_{i} \subset M(\lambda)^{i}$ for some $i \geq 0$. If $\left[M(\lambda)^{i} / M(\lambda)_{i}: L(\mu)\right]=0$ for all $\mu \in H^{*}$ such that $E_{i+1}(M(\lambda)) \cap M(\lambda)_{\mu} \neq$ $E_{i+2}(M(\lambda)) \cap M(\lambda)_{\mu}$, then $M(\lambda)_{i+1} \subset M(\lambda)^{i+1}$. Here $M(\lambda)_{\mu}$ is the $\mu$-weight space of $M(\lambda)$.

The condition as required by the corollary seems hard to come by at this point. However, it would not be too surprising to see that it fits in many interesting cases.

Not only does Theorem 1.2 imply the heredity of the Jantzen filtration for embeddings of Verma modules of this sort, but also our method applies to the relative filtration (the filtration for quotients of Verma modules) to yield an analogous result. From these, one easily deduces the heredity along this line as well.

2. The nondegenerate form on $M(\lambda)^{i} / M(\lambda)^{i+1}$. In this section, we briefly recall the construction of the Jantzen filtration of Verma modules and prove a useful lemma using the associated nondegenerate form on each $M(\lambda)^{i} / M(\lambda)^{i+1}$.

Let $\sigma$ be the unique involutive antiautomorphism of $U(G)$ satisfying $\sigma\left(e_{i}\right)=f_{i}$, $\sigma\left(f_{i}\right)=e_{i}, i=1, \ldots, n$, and $\left.\sigma\right|_{H}=\mathrm{id}$. Following [5], let $A$ be the Shapovalov form on $U(G)$ i.e., $A(x, y)=\operatorname{pr}(\sigma(x) y) \forall x, y \in U(G)$, where $\operatorname{pr}: U(G) \rightarrow U(H)$ is the projection map parallel to the second summand of the decomposition $U(G)=$ $U(H) \oplus\left(N_{-} U(G)+U(G) N_{+}\right)$. For each $\lambda \in H^{*}$, the form $A$ followed by the evaluation at $\lambda$ (under the natural identification $H \leftrightarrow H^{* *}$ ) defines a symmetric bilinear form $A_{\lambda}$ on the Verma module $M(\lambda)$.

Fix an element $\rho \in H^{*}$ satisfying $\rho\left(h_{i}\right)=1 \forall i=1, \ldots, n$. Consider the polynomial ring $\phi[t]$ over $\phi$ on one indeterminate $t$. Let $B_{+}=N_{+} \oplus H$. For each $\lambda \in H^{*}$, let $\phi[t]_{\lambda}=\phi[t]$ (as a set) be the $B_{+}$-module defined by the action $N_{+} \cdot f(t)=0$ and $h \cdot f(t)=\lambda(h) f(t)+\rho(h) t f(t) \forall f(t) \in \phi[t]$. Let $\bar{M}(\lambda)=U(G) \otimes_{U\left(B_{+}\right)} \phi[t]_{\lambda}$ be the induced module. Note that $\bar{M}(\lambda)$ is naturally a $(U(G), \phi[t])$-bimodule. By a trivial extension of the form $A$ on $U(G)$, one defines in the same manner a $(U(H) \otimes \phi[t])$ valued form $\bar{A}$ on $U(G) \otimes \phi[t]$. Followed by "the evaluation at $\lambda+t \rho$ ", one likewise obtains a $\phi[t]$-valued symmetric bilinear form $\bar{A}_{\lambda}$ on each $\bar{M}(\lambda)$. Note that

$$
A_{\lambda}(x \cdot f(t), y)=\bar{A}_{\lambda}(x, y \cdot f(t))=f(t) \bar{A}_{\lambda}(x, y) \quad \forall x, y \in \bar{M}(\lambda) .
$$

Now one obtains for $\bar{M}(\lambda)$ a filtration $\bar{M}(\lambda)=\bar{M}(\lambda)^{0} \supset \bar{M}(\lambda)^{1} \supset \bar{M}(\lambda)^{2} \supset \cdots$ by $(U(G), \phi[t])$-bimodules by setting

$$
\bar{M}(\lambda)^{i}=\left\{x \in \bar{M}(\lambda) \mid \bar{A}_{\lambda}(x, y) \in t^{i} \phi[t] \forall y \in \bar{M}(\lambda)\right\}, \quad i=0,1,2, \ldots
$$

Using the $G$-epimorphism $\pi: \bar{M}(\lambda) \rightarrow M(\lambda)$ given by $t \rightarrow 0$, one obtains for $M(\lambda)$ the Jantzen filtration $M(\lambda)=M(\lambda)^{0} \supset M(\lambda)^{1} \supset M(\lambda)^{2} \supset \cdots$ by letting $M(\lambda)^{i}=$ $\pi\left(\bar{M}(\lambda)^{i}\right), i=0,1,2, \ldots$

For each $i=0,1,2, \ldots$, there is a symmetric bilinear form $\bar{A}_{\lambda}^{i}$ on $\bar{M}(\lambda)^{i}$ defined by $\bar{A}_{\lambda}^{i}(x, y)=\left.\left\{\bar{A}_{\lambda}(x, y) t^{-i}\right\}\right|_{t=0} \forall x, y \in \bar{M}(\lambda)^{i}$. It can be shown that the radical 
of the form $\bar{A}_{\lambda}^{i}$ is given by $\bar{M}(\lambda)^{1}$ if $i=0$ and $\bar{M}(\lambda)^{i-1} \cdot t+\bar{M}(\lambda)^{i+1}$ for $i \geq 1$. Consequently, each $M(\lambda)^{i}$ inherits a symmetric bilinear form $A_{\lambda}^{i}$ whose radical is just $M(\lambda)^{i+1}$. To summarize, we have

Proposition 2.1. For each $i=0,1,2, \ldots, M(\lambda)^{i}$ possesses a symmetric bilinear form $A_{\lambda}^{i}$ satisfying the following properties:

(a) $A_{\lambda}^{i}(u x, y)=A_{\lambda}^{i}(x, \sigma(u) y) \forall x, y \in M(\lambda)^{i}, u \in U(G)$.

(b) $A_{\lambda}^{i}\left(M(\lambda)_{\lambda-\mu}^{i}, M(\lambda)_{\lambda-\nu}^{i}\right)=0$ if $\mu \neq \nu, \mu, \nu \in H^{*}$.

(c) $M(\lambda)^{i+1}$ is the radical of $A_{\lambda}^{i}$.

Proof. (a) and (b) are clear from the construction. (c) can be found in [2].

Conceivably, the existence of these forms $A_{\lambda}^{i}$ is not very demonstrative for the determination of the Jantzen filtration, since, in general, $M(\lambda)^{i}$ has quite a few submodules with respect to which the quotient possesses a nondegenerate form of the same sort. Nevertheless, they can still be effectively used to get useful information under certain specialized circumstances. One such circumstance can be found in the following lemma:

LEMMA 2.2. Let $\lambda \in H^{*}$. Let $i \geq 0, \mu \in H^{*}$ satisfy $\left[M(\lambda)^{i}: L(\mu)\right]=$ $\operatorname{dim} \operatorname{Hom}_{G}\left(M(\mu), M(\lambda)^{i}\right)$. Then $E_{1}\left(M(\lambda)^{i}\right)_{\mu} \subset M(\lambda)^{i+1}$. (Here $E_{1}\left(M(\lambda)^{i}\right)_{\mu}$ is the $\mu$-weight space of $E_{1}\left(M(\lambda)^{i}\right)$, considered as an $H$-module.)

ProOF. Let $M(\lambda)^{i}=N_{0} \supset N_{1} \supset \cdots \supset N_{p}=\{0\}$ be a local composition series of $M(\lambda)^{i}$ at $\mu$ (cf. [1] for the definition). Put $A=\left\{j \mid N_{j} / N_{j+1}\right.$ is irreducible $\}$ and $B=\left\{j \in A \mid N_{j} / N_{j+1} \cong L(\mu)\right\}$. For each $j \in A$, pick a highest weight vector $v_{j}+$ $N_{j+1}$ of $N_{j} / N_{j+1}$. Since we assume $\left[M(\lambda)^{i}: L(\mu)\right]=\operatorname{dim} \operatorname{Hom}_{G}\left(M(\mu), M(\lambda)^{i}\right)$, each $v_{j}$ can be chosen from $E_{0}(M(\lambda))_{\mu}$ if $j \in B$.

Let $y \in\left(M(\lambda)^{i}\right)_{\mu}$. By the nature of the above local composition series, there exist $u_{j} \in U\left(N_{-}\right), j \in A$, such that $y=\sum_{j \in A} u_{j} v_{j}$. Let $x \in E_{1}\left(M(\lambda)^{i}\right)_{\mu}$. If $j \in A-B, u_{j} \in U\left(N_{-}\right)^{0}$, and we have $A_{\lambda}^{i}\left(x, u_{j} v_{j}\right)=A_{\lambda}^{i}\left(\sigma\left(u_{j}\right) x, v_{j}\right)=0$, since $N_{+} x=0$. On the other hand, $j \in B$ implies $u_{j}$ is a constant and we also have $A_{\lambda}^{i}\left(x, u_{j} v_{j}\right)=0$, since $N_{+} v_{j}=0$ and $x \in E_{1}\left(M(\lambda)^{i}\right)_{\mu} \subset U\left(N_{-}\right)^{0} E_{0}\left(M(\lambda)^{i}\right)$. From these we get $A_{\lambda}^{i}(x, y)=0$ for all $y \in\left(M(\lambda)^{i}\right)_{\mu}$. By 2.1, $x \in \operatorname{rad} A_{\lambda}^{i}=M(\lambda)^{i+1}$. This proves the lemma.

3. Proof of Theorem 1.2. We now prove Theorem 1.2 as stated in the introduction. For $\mu, \nu \in H^{*}, \nu \geq \mu$ means $\nu-\mu$ is a nonnegative integral combination of $\alpha_{i}$ 's.

Proof OF THEOREM 1.2. First suppose $M(\lambda)^{i}=M(\lambda)_{i}$ for all $i \geq 0$. Fix $\mu \in H^{*}$. For each $i \geq 0$ and $\nu \in H^{*}$, put $S_{i}(\nu)=U\left(N_{-}\right) E_{0}\left(M(\lambda)_{i}\right)_{\nu}$. Clearly, $M(\lambda)^{i}=M(\lambda)_{i}=\sum_{\nu \in H^{*}} S_{i}(\nu)$ for all $i$. Hence $M(\lambda)_{i} / M(\lambda)_{i+1}$ is a homomorphic image of $\bigoplus_{\nu \in H^{*}} S_{i}(\nu) / \bigoplus_{\nu \in H^{*}} S_{i+1}(\nu)$, and we have

$$
\left[M(\lambda)_{i} / M(\lambda)_{i+1}: L(\mu)\right] \leq \sum_{\nu \in H^{*}}\left[S_{i}(\nu) / S_{i+1}(\nu): L(\mu)\right] \quad \text { for all } i \geq 0 .
$$


Case 1. $\left[S_{i}(\nu) / S_{i+1}(\nu): L(\mu)\right]=0$ for all $i \geq 0$ and $\nu \neq \mu$. In this case,

$$
\begin{aligned}
{[M(\lambda): L(\mu)] } & =\sum_{i \geq 0}\left[M(\lambda)_{i} / M(\lambda)_{i+1}: L(\mu)\right] \leq \sum_{i \geq 0}\left[S_{i}(\mu) / S_{i+1}(\mu): L(\mu)\right] \\
& =\sum_{i \geq 0}\left(\left[S_{i}(\mu): L(\mu)\right]-\left[S_{i+1}(\mu): L(\mu)\right]\right)=\left[S_{0}(u): L(\mu)\right] \\
& =\operatorname{dim} E_{0}(M(\lambda))_{\mu}=\operatorname{dim} \operatorname{Hom}_{G}(M(\mu), M(\lambda)) .
\end{aligned}
$$

The result is clear since the reverse inequality always holds.

Case 2. There exists $i \geq 0$ and $\nu>\mu$ such that $\left[S_{i}(\nu) / S_{i+1}(\nu): L(\mu)\right] \neq 0$. We show that this case cannot happen.

Under the above assumption, it is easy to see that there exists $x \in E_{0}\left(M(\lambda)_{i}\right)_{\nu}$ such that $\left[U(G) x / U(G) x \cap M(\lambda)_{i+1}: L(\mu)\right] \neq 0$. Let $u \in U\left(N_{-}\right)_{\mu-\nu}$ satisfy $u x \notin M(\lambda)_{i+1}$ and $U(G) u x / N \cong L(\mu)$ where $N$ is a maximal submodule of $U(G) u x$. Note that $\sigma\left(u^{\prime}\right) u x=0$ for all $u^{\prime} \in U\left(N_{-}\right)_{\mu-\nu}$, since $x \notin N$. Let $y \in$ $\left(M(\lambda)^{i}\right)_{\mu}$. Since $M(\lambda)^{i}=M(\lambda)_{i}, y \in U\left(N_{-}\right) E_{i}(M(\lambda))$. Let $y=\sum_{j} u_{j} x_{j}$ where $x_{j} \in E_{i}(M(\lambda))_{\nu_{j}}, u_{j} \in U\left(N_{-}\right)$. If $v_{j}<\nu, A_{\lambda}^{i}\left(u_{j} x_{j}, u x\right)=A_{\lambda}^{i}\left(\sigma(u) u_{j} x_{j}, x\right)=0$, since $N_{+} x_{j}=0$. On the other hand, $A_{\lambda}^{i}\left(u_{j} x_{j}, u x\right)=A_{\lambda}^{i}\left(x_{j}, \sigma\left(u_{j}\right) u x\right)=0$ if $v_{j} \geq \nu$ since $\sigma\left(u_{j}\right) u x=0$. This implies $A_{\lambda}^{i}(y, u x)=0 \forall y \in\left(M(\lambda)^{i}\right)_{\mu}$. Hence $u x \in \operatorname{rad} A_{\lambda}^{i}=M(\lambda)^{i+1}=M(\lambda)_{i+1}$, a contradiction. So this case can not happen. This proves that $[M(\lambda): L(\mu)]=\operatorname{dim} \operatorname{Hom}_{G}(M(\mu), M(\lambda))$ for all $\mu \in H^{*}$.

Conversely, let us suppose $[M(\lambda): L(\mu)]=\operatorname{dim}_{\operatorname{Hom}}(M(\mu), M(\lambda))$ for all $\mu \in$ $H^{*}$. It is easy to see that this assumption implies that

$$
[N: L(\mu)]=\operatorname{dim} \operatorname{Hom}_{G}(M(\mu), N)
$$

for all submodules $N$ of $M(\lambda)$. In particular, if $x \in E_{i}(M(\lambda))_{\nu}$ for some $\nu \in H^{*}$, $U(G) x \cap M(\lambda)_{i+1}$ is either equal to $U(G) x$ or equal to the maximal submodule of $U(G) x$ according as $x \in E_{i+1}(M(\lambda))_{\nu}$ or not.

Using Lemma 2.2 inductively, one easily obtains $M(\lambda)_{i} \subset M(\lambda)^{i}$ for all $i \geq 0$. To prove the result by contradiction, let $j$ be the smallest integer satisfying $M(\lambda)_{j} \varsubsetneqq$ $M(\lambda)^{j}$. Clearly $j \geq 1$. Let $x \in M(\lambda)^{j}-M(\lambda)_{j}$.

As previously defined, $M(\lambda)^{j-1}=M(\lambda)_{j-1}=\sum_{\nu \in H^{*}} S_{j-1}(\nu)$. Since $M(\lambda)^{j} \subset$ $M(\lambda)^{j-1}, x=\sum_{\nu \in H^{*}} x_{\nu}$ where $x_{\nu} \in S_{j-1}(\nu)$. Now, by an earlier comment, $S_{j-1}(\nu) \cap M_{j}(\lambda)$ contains the maximal submodule of each $U(G) y, y \in E_{j-1}(M(\lambda))_{\nu}$. In other words, $S_{j-1}(\nu) / S_{j-1}(\nu) \cap M(\lambda)_{j}$ is isomorphic to a direct sum of $L(\nu)$ 's. So, if $\nu_{0}$ is a maximal element in $H^{*}$ such that $x_{\nu_{0}} \notin M(\lambda)_{j}$ (such a $\nu_{0}$ exists since $\left.x \notin M(\lambda)_{j}\right)$, it is easy to see that $\left\{U(G) x \cap E_{j-1}(M(\lambda))_{\nu_{0}}\right\}-M(\lambda)_{j}$ is not empty. Thus we may as well assume that $x \in E_{j-1}(M(\lambda))_{\nu_{0}}-M(\lambda)_{j}$. In particular, $x \notin U\left(N_{-}\right)^{0} M(\lambda)_{j-1}$.

Since $x \in M(\lambda)^{j}$, there exists $x(t) \in \bar{M}(\lambda)^{j}$ such that $\pi(x(t))=x$. Let $\Delta$ be the set of positive roots of $G$. For each $\alpha \in \Delta$, let $\left\{e_{\alpha}^{1}, e_{\alpha}^{2}, \ldots\right\}$ be a basis for the root space $G_{\alpha}$. Since $x \in E_{j-1}(M(\lambda)) \subset E_{0}(M(\lambda)), N_{+} x=0$. This implies that $N_{+} x(t) \in \bar{M}(\lambda) t$. So we may write $e_{\alpha}^{i} x(t)=x_{\alpha}^{i} \otimes t\left(\bmod \bar{M}(\lambda) t^{2}\right)$. Since $x(t) \in \bar{M}(\lambda)^{j}, x_{\alpha}^{i} \in M(\lambda)^{j-1}$ for all $\alpha, i$.

We now recall the operator $\Omega=\sum_{\alpha \in \Delta} \sum_{i} e_{-\alpha}^{i} e_{\alpha}^{i}$ where $\left\{e_{-\alpha}^{1}, e_{-\alpha}^{2}, \ldots\right\}$ is the dual basis of $\left\{e_{\alpha}^{1}, e_{\alpha}^{2}, \ldots\right\}$ in $G_{-\alpha}$ relative to an invariant bilinear form, as we assume $G$ is symmetrizable. It is known that $\Omega$ acts on $\bar{M}(\lambda)_{\lambda-\nu}=U\left(N_{-}\right)_{-\nu} \otimes \phi[t]$ as 
the scalar $(\nu, \rho) t$ for all $\nu \in H^{*}$ such that $E_{0}(M(\lambda))_{\lambda-\nu} \neq\{0\}$ (cf. [3]). Thus $\Omega \cdot x(t)=\left(\lambda-\nu_{0}, \rho\right) x(t) t=\sum_{\alpha \in \Delta} \sum_{i} e_{-\alpha}^{i} x_{\alpha}^{i} \otimes t\left(\bmod \bar{M}(\lambda) t^{2}\right)$. This implies that $\left(\lambda-\nu_{0}, \rho\right) x=\sum_{\alpha \in \Delta} \sum_{i} e_{-\alpha}^{i} x_{\alpha}^{i}$. Note that $\left(\lambda-\nu_{0}, \rho\right) \neq 0$ since $\nu_{0} \neq \lambda$. Hence $x \in U\left(N_{-}\right)^{0} M(\lambda)^{j-1}=U\left(N_{-}\right)^{0} M(\lambda)_{j-1}$, a contradiction. This proves $M(\lambda)^{i}=M(\lambda)_{i}$ for all $i \geq 0$.

The proof of the theorem is complete.

REMARK 3.1. For nonsymmetrizable Kac-Moody Lie algebras, $M(\lambda)_{i}=M(\lambda)^{i}$ $\forall i \geq 0 \Rightarrow[M(\lambda): L(\mu)]=\operatorname{dim}_{\operatorname{Hom}_{G}}(M(\mu), M(\lambda)) \forall \mu \in H^{*}$ and $[M(\lambda): L(\mu)]=$ $\operatorname{dim} \operatorname{Hom}_{G}(M(\mu), M(\lambda)) \forall \mu \in H^{*} \Rightarrow M(\lambda)_{i} \subseteq M(\lambda)^{i} \forall i \geq 0$.

As one can see now, Corollary 1.3 may be proved using the same technique. Another immediate consequence is the following corollary concerning the heredity of the Jantzen filtration for embeddings.

Corollary 3.2. Let $G$ be symmetrizable, $\lambda \in H^{*}$. Suppose $[M(\lambda): L(\mu)]=$ $\operatorname{dim} \operatorname{Hom}_{G}(M(\mu), M(\lambda))$ for all $\mu \in H^{*}$. If $v \in E_{i}(M(\lambda))_{\mu}-E_{i+1}(M(\lambda))_{\mu}$ for some $\mu \in H^{*}, i>0$, and $N=U\left(N_{-}\right) v \cong M(\mu)$, then $N=N \cap M(\lambda)^{i} \supset N \cap M(\lambda)^{i+1} \supset$ $N \cap M(\lambda)^{i+2} \supset \cdots$ is the Jantzen filtration of $N$.

ProOF. This is evident from Theorem 1.2 since $N \cap M(\lambda)^{i+k}=N \cap M(\lambda)_{i+k}=$ $N_{k}$ for all $k$.

REMARK 3.3. Following Jantzen [2], in general, it may be conjectured that Corollary 3.2 holds for arbitrary Verma modules.

Since a complete analogue of Proposition 2.1 exists for the relative filtration, our method also applies along this line. The result may be stated as follows (for the construction of the relative filtration, consult with $[\mathbf{2}, \mathbf{4}])$.

COROLlaRY 3.4. Let $G$ be symmetrizable, $\lambda \in H^{*}$. Suppose $\alpha$ is a positive real root of $G$ and $M(\lambda-k \alpha)$ can be embedded in $M(\lambda)$ for some $k \in N$. Let $S \cong M(\lambda-k \alpha)$ be the embedding of $M(\lambda)$ defined by the Shapovalov element. Then $M(\lambda) / S \supset M(\lambda)^{1}+S / S \supset M(\lambda)^{2}+S / S \supset \cdots$ is the relative filtration of the quotient $M(\lambda) / S$, provided that

$$
[M(\lambda): L(\mu)]=\operatorname{dim} \operatorname{Hom}_{G}(M(\mu), M(\lambda)) \quad \forall \mu \in H^{*} .
$$

Proof. Mimic the proof of Theorem 1.2 and use the fact that $(M(\lambda) / S)_{i}=$ $M(\lambda)_{i}+S / S \forall i \geq 0$, under the assumption.

REMARK 3.5. Following Jantzen [2], in general, it may be conjectured that Corollary 3.4 holds for arbitrary Verma modules.

REMARK 3.6. Known examples of Verma modules satisfying the assumption of Theorem 1.2 are those associated with rank two Weyl groups and $M(-\rho)$ over Euclidean Lie algebras.

\section{REFERENCES}

1. V. V. Deodhar, O. Gabber, and V. G. Kac, Structure of some categories of representations of infinite dimensional Lie algebras, Adv. in Math. 45 (1982), 91-116.

2. J. C. Jantzen, Modulen mit einem höchsten Gewicht, Lecture Notes in Math., vol. 750, Springer-Verlag, Berlin and New York, 1979.

3. V. G. Kac and D. A. Kazhdan, Structure of representations with highest weight of infinite dimensional Lie algebras, Adv. in Math. 34 (1979), 97-108. 
4. A. Rocha-Caridi and N. R. Wallach, Highest weight modules over infinite dimensional graded Lie algebras: resolutions, filtrations and character formulas, Trans. Amer. Math. Soc. 277 (1983), 133-162.

5. N. N. Shapovalov, On a bilinear form on the universal enveloping algebra of a complex semisimple Lie algebra, Funct. Anal. Appl. 6 (1972), 307-312.

Department of Mathematics, Ohio State University, Columbus, Ohro 43210

Current address: Institute of Mathematics, Academia Sinica, Nankang, Taipei, Taiwan 11529, Republic of China 